\title{
The role of angiotensin-converting enzyme gene insertion/deletion polymorphism for blood pressure regulation in areca nut chewers
}

\author{
FU-MEI CHUNG, TIEN-YU SHIEH, YI-HSIN YANG, DAO-MING CHANG, SHYI-JANG SHIN, \\ JACK C-R TSAI, TONY HSIU-HSI CHEN, TONG-YUAN TAI, and YAU-JIUNN LEE
}

PINGTUNG, KAOHSIUNG, AND TAIPEI, TAIWAN

\begin{abstract}
Areca tannin has been suggested as having a blood pressure regulatory effect through its ability to inhibit the pressor response to both angiotensin I and II. As genetic and environmental factors determine the susceptibility and development of diseases and no report has been published concerning the genetic interaction of metabolic effects in areca nut/betel quid $(B Q)$ chewers, it is proposed that the cardiovascular effects of chronic $B Q$ usage can be affected by the polymorphism of the angiotensin converting enzyme (ACE) gene. This is a cross-sectional community-based study. A total of $189 \mathrm{BQ}$ chewing subjects and 256 non-chewing controls were studied. $\mathrm{BQ}$ chewing status was determined by using a structured questionnaire, and insertion/deletion (I/D) polymorphisms were determined by a polymerase chain reaction. BQ chewers with the DD genotype had significantly lower blood pressure, pulse pressure, and prevalence of hypertension compared with those of chewers with II or ID genotypes. Multiple stepwise regression analysis confirmed that the ACE I/D genotype was independently associated with systolic blood pressure (SBP). BQ chewers with the DD genotype accounted for a significant age, sex, and waist-to-hip ratio adjusted decrease in SBP $(-5.9 \mathrm{~mm} \mathrm{Hg}, P=0.021)$. This finding suggests that $B Q$ chewing may be related to blood pressure regulation, which supports the hypothesis that concomitant genetic susceptibility and environmental factors determine the level of blood pressure. (Translational Research 2007; 150:58-65)
\end{abstract}

\begin{abstract}
Abbreviations: $\mathrm{ACE}=$ angiotensin converting enzyme; $\mathrm{ACEI}=\mathrm{ACE}$ inhibitor; $\mathrm{ANOVA}=$ analysis of variance; $\mathrm{BMI}=$ body mass index; $\mathrm{BQ}=$ betel quid; $\mathrm{DBP}=$ diastolic blood pressure; $\mathrm{GOT}=$ glutamic oxaloacetic transferase; $\mathrm{HDL}=$ high-density lipoprotein; I/D = insertion/ deletion; LDL = low-density lipoprotein; SBP = systolic blood pressure; WHR = waist-to-hip ratio
\end{abstract}

A reca nut/betel quid (BQ; Areca catechu) is said to be the fourth most commonly used psychoactive substance in the world, after caffeine, nicotine, and alcohol, and it is chewed regularly by at least $10 \%$ of the world's population. ${ }^{1}$ It is estimated that 2

From the Department of Clinical Research, Pingtung Christian Hospital, Pingtung, Taiwan; the Graduate Institute of Dental Sciences, College of Dental Medicine, the Graduate Institute of Oral Health Sciences, and the Graduate Institute of Medicine, Kaohsiung Medical University, Kaohsiung, Taiwan; the Institute of Preventive Medicine, College of Public Health, National Taiwan University, and the Department of Internal Medicine, Ren-Ji Hospital, Taipei; and Lee's Endocrinologic Clinic, Pingtung, Taiwan.

Supported by grants from the National Science Council (95-2314-B- million persons in Taiwan have the habit of chewing BQ, which is especially common among aborigines. The prevalence may be up to $50 \%$ in women. ${ }^{2,3}$ Many people have acquired the habit because of its physiologic effects, in-

475-002-MY3 and 95-2314-B-037-056) and the Department of Health of Taiwan (DOH92-HP-1111).

Submitted for publication April 24, 2006; revision submitted January 11, 2007; accepted for publication January 20, 2007.

Reprint requests: Yau-Jiunn Lee, Lee's Endocrinologic Clinic, No. 130 Min-Tsu Road, Pingtung, 90000 Taiwan; e-mail: t3275@ms25.hinet.net. $1931-5244 / \$$ - see front matter

(c) 2007 Mosby, Inc. All rights reserved.

doi:10.1016/j.trs1.2007.01.005 
cluding increased stamina and a general feeling of wellbeing. ${ }^{1}$ Both benign and malignant diseases can be induced by BQ chewing, with deleterious effects on the oral soft tissues, which has been published extensively in the dental literature. ${ }^{4}$ Reports have shown that chronic BQ chewing is associated with diverse systemic effects, including hyperglycemia, insulin resistance, obesity, cardiorespiratory disease, and dependence syndrome ${ }^{5-10} \mathrm{Re}$ cently, it was observed that chronic BQ use is associated with a higher risk of type 2 diabetes mellitus and metabolic syndrome, determined by a epidemiologic survey in Taiwan. ${ }^{11,12}$

The major compounds of BQ are polyphenolic compounds, alkaloids, tannin, and fibers. ${ }^{13}$ Interestingly, areca tannin has been suggested to have properties as a hypotensive drug through its ability to inhibit the pressor response to both angiotensin I and II. ${ }^{14}$ Acute cardiovascular responses occur, such as increased heart rate and blood pressure in new BQ chewers, whereas no significant effects on blood pressure have been observed in chronic BQ chewers. ${ }^{15}$

The angiotensin converting enzyme (ACE) plays a key role in modulating vascular tone and electrolyte balance by hydrolyzing angiotensin I to angiotensin II, which is a potent vasopressor and aldosterone-stimulating peptide. ${ }^{16}$ The individual variation of plasma ACE activity is largely affected by polymorphism of the $A C E$ gene. ${ }^{17}$ Specifically, $A C E$ gene I/D polymorphism has been found to be associated, albeit somewhat controversially, with the development of hypertension and the response of ACE inhibitor (ACEI) treatment in patients with essential hypertension. ${ }^{18-20}$

In a recent report by the authors, it was observed that $A C E$ insertion/deletion (I/D) polymorphism is associated with the risk of oral mucosal lesions in BQ chewers ${ }^{21}$ which indicates the relative contribution of genetic and environmental factors that determine the susceptibility and development of diseases. As no report has been published concerning the genetic interaction of metabolic effects in BQ chewers, we propose that the cardiovascular and metabolic effects of chronic BQ usage may be affected by the polymorphism of the $A C E$ gene. To test this hypothesis, the role of the $A C E$ gene I/D polymorphism has been investigated in the systemic effects of BQ chewing in an aboriginal community with a high prevalence of BQ chewing habit.

\section{MATERIALS AND METHODS}

Subjects. All data were derived from the Aboriginal Health Interview and Integrated Health Care Program for rural indigenous people over 30 years old, which was conducted by the Bureau of National Health Insurance of Taiwan from January 2000 to December 2003 as recently reported. ${ }^{21,22}$ Using population registries, this study selected a stratified random sample of 1466 subjects, representative for age, gender, and aboriginal community. The overall participation rate was 59\% according to the 2002 Official Household Registry. Subjects with hypertension were defined as having a systolic blood pressure $(\mathrm{SBP}) \geq 140 \mathrm{~mm} \mathrm{Hg}$, diastolic blood pressure (DBP) $\geq 90 \mathrm{~mm} \mathrm{Hg}$, or both, or as receiving antihypertensive medication indicated for treatment of hypertension. For the purposes of the current study, 84 participants were excluded with known major systemic diseases (eg, coronary heart disease, hypertrophic cardiomyopathy, liver cirrhosis, renal insufficiency, and malignancies). Subjects of 813 areca nut chewers who smoked or consumed alcohol were excluded to avoid interfering effects on the metabolic measurements. Also, 124 participants were excluded for other reasons, including 58 persons with an age $<40$ years old, 34 persons with residence outside aboriginal communities, and 32 persons with missing information. This study was approved by the Human Research Ethics Committee of the participating hospital, and informed consent was obtained from each participant.

All study subjects lived in the same region at the time of the study and were of Pai-Wan ethnicity without known ancestry from other ethnicities. Each subject was personally interviewed with regard to demographic information, occupation, physical activity, BQ chewing, smoking history, alcohol drinking habits, as well as personal and family history of various diseases using a structured questionnaire. ${ }^{21}$ The physical activity of the study subjects was evaluated by 2 types of exercise status: nonexercisers and moderate exercisers. The main varieties of chews used by the aborigines studied were those prepared by combining unripe areca fruit with a piece of inflorescence (flower head) of the Piper betle vine and red lime paste or by wrapping unripe areca fruit in leaves of the Piper betle vine with white lime paste. ${ }^{3}$ It should be noted that no tobacco was added to the chews used in Taiwan. Subjects who had chewed 1 BQ or more per day for least 1 year were defined as those having chewed. Among them, current chewers were those who practiced this habit within the previous 1 year, and ex-chewers were those who had stopped for less than 1 year before the interview. In this study, current chewers and ex-chewers were analyzed as a group and compared with those who had never been chewers and those who had chewed before who had stopped the habit for at least 1 year. For all of those who had chewed before, the duration of BQ use and the amount of BQ chewed each day were also recorded. The cumulative exposure to BQ chewing was derived by multiplying the daily consumption of BQ chewing (in quid/day) by the duration of BQ chewing (in years).

Venous blood samples were drawn for biochemical analyses after an overnight fasting period of at least $8 \mathrm{~h}$. The plasma biochemical parameters were measured after overnight fasting as previously described. ${ }^{23}$ Data on the biological factors associated with the risk of hypertension were collected, including plasma triglycerides, total cholesterol, lowdensity lipoprotein (LDL)-cholesterol, high-density lipoprotein (HDL)-cholesterol, uric acid, creatinine, glucose, and glutamic oxaloacetic transferase (GOT). Body height, weight, and waist and hip circumferences were measured, and the body mass index (BMI) and waist-to-hip ratio (WHR) were 
calculated. Blood pressure was measured in the morning (readings were taken twice, at least 2 min apart), on the right upper arm in the line of the heart, in a sitting position after a minimum of 5 min of rest. BP was read by a mercury column sphygmomanometer. The average value of these measurements was used for analysis.

ACE I/D polymorphism. Genomic DNA was prepared from peripheral blood using standard techniques. For the $A C E$ I/D polymorphism, the primer pairs used and the annealing temperatures were as follows: forward 5'-CTGGAGACCACTCCCATCCTTTCT-3' and reverse 5'-GATGTGGCCATCACATTCGTCAGAT-3', which amplify the intron 16 region where the I/D fragment is located. ${ }^{24}$ To avoid ID/DD mistyping of the heterozygote as DD-homozygote, ${ }^{25}$ all DDgenotype samples were confirmed using a pair of primers that produced an amplified product only in the presence of the insertion and was used to verify the polymorphism: forward, 5'-TGGGACCACAGCGCCCGCCACTAC-3' and reverse, 5'-TCGCCAGCCCTCCCATGCCCATAA-3'. All PCR products were visualized after electrophoresis on a $2 \%$ agarose gel and ethidium bromide staining. To assure that the genotyping was of sufficient quality, we performed random duplicates in more than $10 \%$ of the samples and we carried controls from carriers and noncarriers in each genotyping assay. The assays were performed by a technician who was blind to the phenotype. No genotype errors were detected in the random duplicates.

Statistical analysis. Descriptive data were examined for all variables. For continuous variables, results were presented as the mean $\pm \mathrm{SD}$, geometric mean (1-SD interval), or median (interquartile range) when appropriate. Statistical differences in variables were compared using unpaired Student $t$-tests and 1-way analysis of variance (ANOVA) for normally distributed variables, followed by Turkey's pair-wise comparison. Categorical variables were recorded as frequency counts, and intergroup comparisons were analyzed by the $\chi^{2}$-test. General linear modeling function analysis was used to control for potential confounders other than age (eg, sex, BMI, WHR, total cholesterol and HDL-cholesterol levels, use of antihypertensive medication, diabetes, and physical activity status).

Associations between systolic blood pressure and other parameters by BQ chewing were first analyzed by simple linear regression and then by multiple stepwise regression analysis. Age, gender, BMI, WHR, fasting glucose, totalcholesterol, triglyceride, HDL-cholesterol, LDL-cholesterol, uric acid, creatinine, GOT, and ACE gene I/D genotypes were included in the analysis. The interactions between BQ chewing and $A C E$ genotypes were assessed using a cross-product term between genotypes and BQ chewing status. Statistical significance was accepted if $P<0.05$. All statistical analysis was performed using SAS statistical software, version 8.2 (SAS Institute Inc., Cary, NC).

\section{RESULTS}

Data were collected from a total of 1466 rural indigenous people, of which 1021 persons for reasons listed in the subjects and methods section were excluded. A total of 189 BQ chewing subjects without alcohol drinking and smoking habits were selected as case subjects, and 256 without any BQ chewing, drinking, and smoking habits were used as controls. None of the study subjects had coffee consumption habits.

Table I shows the clinical characteristics of the study subjects grouped by their BQ usage status. BQ-chewing subjects had female predominance, higher BMI, WHR, and serum triglyceride levels and lower creatinine and uric acid levels than nonchewers. The systolic and diastolic BPs, fasting plasma glucose, serum total cholesterol, HDL- and LDL-cholesterol, GOT levels, and the prevalence of hypertension, diabetes, physical activity status, and antihypertensive drug use were not statistically different between the study groups. The mean duration of BQ chewing in the case group was 34 years.

Table II presents the general characteristics of the 2 study groups stratified by $A C E$ I/D genotypes with ANOVA, followed by Turkey's pair-wise comparison and the $\chi^{2}$-test. In all study subjects, the genotype distributions of this polymorphism were in HardyWeinberg equilibrium. Within the non-BQ chewing group, no significant differences in general characteristics were observed among the genotypes. The prevalence of hypertension, SBP, and pulse pressure in BQ chewers with DD genotypes was significantly lower compared with those with ID and II genotypes even after adjustment for age, sex, BMI, WHR, total cholesterol, HDL-cholesterol, use of antihypertensive medication, diabetes, and physical activity status. The SBP and prevalence of hypertension of BQ chewers with the II genotype $(150 \pm 22 \mathrm{~mm} \mathrm{Hg}$ and $68 \%$, respectively) were different from those of nonchewers (134 \pm 23 $\mathrm{mm} \mathrm{Hg}$ and $52.6 \%$, respectively) with the II genotype, whereas the difference was not statistically significant after adjusting by general linear models for age, sex, BMI, WHR, total cholesterol, HDL-cholesterol, use of antihypertensive drugs, diabetes, and physical activity status.

Simple linear regression analysis has revealed that age, BMI, fasting glucose, triglyceride, creatinine, and the $A C E \mathrm{I} / \mathrm{D}$ genotype are associated with SBP in $\mathrm{BQ}$ chewers. Age, WHR, uric acid, and creatinine are associated with SBP in nonchewers. Multiple stepwise regression analysis confirmed that age, fasting glucose, and the $A C E \mathrm{I} / \mathrm{D}$ genotype are independent risk factors for SBP in BQ chewers, whereas age and BMI are factors for SBP in nonchewers (Table III). The interaction between the $A C E$ I/D genotype and BQ chewing in relation to $\operatorname{SBP}(P$ value for interaction $=0.006)$ is statistically significant.

Figure 1 shows the prevalence of hypertension stratified by $A C E$ genotypes in BQ chewers and nonchewers. Within the chewers group, the hypertension rate 
Table I. Clinical and biochemical characteristics of the study subjects

\begin{tabular}{|c|c|c|c|c|}
\hline Variable & All subjects $(\mathrm{N}=445)$ & $B Q$ chewers $(N=189)$ & Non- $B Q$ chewers $(N=256)$ & $P$ value \\
\hline Age (years) & $61.5 \pm 13.0$ & $62.4 \pm 12.9$ & $60.8 \pm 13.0$ & 0.186 \\
\hline Sex, male, (\%) & 25.2 & 15.3 & 32.4 & $<0.0001$ \\
\hline $\mathrm{BMI}\left(\mathrm{kg} / \mathrm{m}^{2}\right)$ & $27.1 \pm 4.9$ & $27.7 \pm 5.4$ & $26.6 \pm 4.4$ & 0.031 \\
\hline WHR & $0.87 \pm 0.08$ & $0.88 \pm 0.09$ & $0.86 \pm 0.07$ & 0.041 \\
\hline Hypertension (\%) & 51.9 & 52.7 & 51.3 & 0.776 \\
\hline Diabetes mellitus (\%) & 14.0 & 14.4 & 13.7 & 0.818 \\
\hline Antihypertensive drug use (\%) & 25.2 & 25.4 & 25.0 & 0.923 \\
\hline Systolic BP (mmHg) & $136 \pm 22$ & $138 \pm 22$ & $135 \pm 22$ & 0.186 \\
\hline Diastolic BP (mmHg) & $81 \pm 12$ & $81 \pm 12$ & $80 \pm 12$ & 0.396 \\
\hline Fasting glucose (mmol/L) & $6.1 \pm 2.7$ & $6.1 \pm 2.5$ & $6.1 \pm 2.8$ & 0.785 \\
\hline T-cholesterol (mmol/L) & $5.08 \pm 0.89$ & $5.07 \pm 0.89$ & $5.08 \pm 0.89$ & 0.915 \\
\hline Triglyceride $(\mathrm{mmol} / \mathrm{L})^{\star}$ & $1.26(0.75-1.47)$ & $1.38(0.80-1.52)$ & $1.17(0.72-1.38)$ & 0.007 \\
\hline HDL-cholesterol (mmol/L) & $1.2 \pm 0.3$ & $1.2 \pm 0.3$ & $1.2 \pm 0.3$ & 0.947 \\
\hline LDL-cholesterol (mmol/L) & $3.20 \pm 0.79$ & $3.14 \pm 0.77$ & $3.24 \pm 0.80$ & 0.213 \\
\hline SGOT (U/L) & $27.8 \pm 10.9$ & $27.6 \pm 9.5$ & $27.9 \pm 11.9$ & 0.804 \\
\hline Creatinine ( $\mu \mathrm{mol} / \mathrm{L})$ & $82.2 \pm 34.5$ & $78.7 \pm 23.0$ & $84.9 \pm 40.7$ & 0.044 \\
\hline Uric acid (mmol/L) & $0.38 \pm 0.09$ & $0.37 \pm 0.08$ & $0.40 \pm 0.10$ & 0.008 \\
\hline Moderate exercise (\%) & 72.8 & 71.3 & 73.8 & 0.627 \\
\hline Betel chewing duration (years) ${ }^{\dagger}$ & - & $34.0(20.0-48.0)$ & - & \\
\hline $\begin{array}{l}\text { Cumulative amount of quid } \\
\text { consumption (quid-years) }\end{array}$ & - & $95,760(43,680-194,544)$ & - & \\
\hline
\end{tabular}

Abbreviation: SGOT, serum glutamic oxaloacetic transferase.

Note: Data are expressed as means $\pm \mathrm{SD}$.

${ }^{*}$ Geometric mean (1-SD interval).

${ }^{\dagger}$ Median (interquartile range). Comparisons between $\mathrm{BQ}$ chewers and non-BQ chewers are performed by unpaired Student $t$-test or $\chi^{2}$-test.

decreased significantly with the number of $\mathrm{D}$ alleles present $(P$ value for trend $<0.05)$. However, in non-BQ chewers, there was no statistical difference in the prevalence of hypertension among subjects with different $A C E \mathrm{I} / \mathrm{D}$ genotypes.

In multiple linear regression analysis using the II genotype as a reference group, the presence of the DD genotype is associated with both SBP $(-5.9 \mathrm{~mm} \mathrm{Hg}$, $P=0.021)$ and pulse pressure $(-5.8 \mathrm{~mm} \mathrm{Hg}, P=$ 0.004 ) in BQ-chewers after adjustment for age, sex, and WHR.

\section{DISCUSSION}

These results demonstrate that BQ chewers with the $A C E$ DD genotype have significantly decreased SBP and a decreased risk of hypertension compared with chewers who carry the I allele. The current study confirmed the recent observation in aborigines that geneticenvironmental factors contribute to the susceptibility and development of diseases in BQ consumption. ${ }^{21}$ This report seems to be the first to demonstrate that $A C E$ I/D genotypes may affect blood pressure homeostasis in BQ chewers.

ACE inhibitor-like activity of the areca nut extract was observed by Inokuchi et al, ${ }^{14}$ and this activity was comparable with that of captopril. The vasopressor response to norepinephrine and vasodepressor responses to bradykinin and acetylcholine were not ap- preciably changed by intravenous treatment with areca extract, although it did produce a dose-related inhibition of the pressor responses to angiotensin I and II. These findings suggest that the areca extract has favorable properties as a hypotensive drug through its ability to inhibit the pressor responses to both angiotensin I and II. ${ }^{14}$ ACE is a key component in the renninangiotensin system, converting angiotensin I to angiotensin II. It also inactivates the vasodilator bradykinin. Both peptides play central roles in blood pressure regulation and are believed to be important in the pathogenesis of cardiovascular diseases. ${ }^{26}$ ACE levels in plasma and tissue are under genetic control. An I/D polymorphism exists in the $A C E$ gene characterized by the presence or absence of a $287 \mathrm{bp}$ alu repeat. Subjects with the DD genotype have higher plasma ACE activity compared with those with ID and II genotypes. ${ }^{17,27}$ In Caucasians, $44 \%$ of the variance in circulating ACE activity was accounted for by genetic polymorphism, ${ }^{27}$ and similar results have been found in the Chinese population. ${ }^{28}$ Subjects with the DD genotype have markedly higher plasma ACE levels than those with genotype II. The ACE gene polymorphism has also been shown to contribute to the differences in response to ACEI treatment. ${ }^{29}$ Among hypertensive patients treated with ACE inhibitors, patients with the D allele of the ACE gene are associated with significantly greater blood pressure reductions. ${ }^{29-32}$ The chewers 
Table II. Variables according to ACE genotypes by betel quid chewing status

\begin{tabular}{|c|c|c|c|c|c|c|c|c|c|}
\hline \multirow[b]{2}{*}{ Variable } & \multicolumn{5}{|c|}{$B Q$ chewers $(n=189)$} & \multicolumn{4}{|c|}{ Non- $B Q$ chewers $(n=256)$} \\
\hline & II & ID & DD & $P$ & $p^{a}$ & II & ID & DD & $P$ \\
\hline Age $(y r)$ & $63.9 \pm 13.4$ & $62.1 \pm 11.8$ & $62.5 \pm 14.2$ & 0.802 & & $59.3 \pm 14.8$ & $63.0 \pm 14.0$ & $62.2 \pm 12.7$ & 0.856 \\
\hline Sex, male, (\%) & 21.4 & 15.1 & 13.0 & 0.580 & & 27.7 & 34.4 & 31.7 & 0.695 \\
\hline $\begin{array}{l}\text { Cumulative amount of quid consumption } \\
\text { (quid-years) }\end{array}$ & $141,500 \pm 165,500$ & $157,700 \pm 263,200$ & $186,100 \pm 234,900$ & 0.694 & & - & - & - & \\
\hline $\mathrm{BMI}\left(\mathrm{kg} / \mathrm{m}^{2}\right)$ & $27.7 \pm 4.5$ & $27.7 \pm 6.0$ & $27.7 \pm 5.0$ & 0.998 & & $26.4 \pm 4.2$ & $26.4 \pm 4.5$ & $27.2 \pm 4.3$ & 0.445 \\
\hline WHR & $0.89 \pm 0.08$ & $0.90 \pm 0.11$ & $0.86 \pm 0.05$ & 0.036 & & $0.88 \pm 0.09$ & $0.85 \pm 0.08$ & $0.87 \pm 0.06$ & 0.120 \\
\hline Hypertension (\%) & 68.0 & 59.3 & 37.3 & 0.009 & 0.035 & 52.6 & 50.4 & 52.0 & 0.962 \\
\hline Systolic BP (mmHg) & $150 \pm 22$ & $136 \pm 23$ & $134 \pm 18$ & 0.007 & 0.044 & $134 \pm 23$ & $134 \pm 22$ & $137 \pm 22$ & 0.639 \\
\hline Diastolic BP (mmHg) & $81 \pm 16$ & $82 \pm 12$ & $81 \pm 12$ & 0.909 & & $81 \pm 12$ & $79 \pm 13$ & $82 \pm 11$ & 0.318 \\
\hline Pulse pressure (mmHg) & $69.1 \pm 19.1$ & $55.7 \pm 17.6$ & $52.7 \pm 13.5$ & 0.0003 & 0.028 & $52.7 \pm 16.8$ & $55.3 \pm 17.9$ & $55.3 \pm 17.4$ & 0.712 \\
\hline Fasting glucose (mmol/L) & $6.1 \pm 3.0$ & $6.0 \pm 2.6$ & $6.1 \pm 2.3$ & 0.962 & & $6.4 \pm 3.9$ & $6.3 \pm 2.9$ & $5.8 \pm 1.7$ & 0.335 \\
\hline T-cholesterol (mmol/L) & $5.04 \pm 0.91$ & $5.14 \pm 0.92$ & $5.01 \pm 0.85$ & 0.627 & & $5.29 \pm 0.83$ & $4.97 \pm 0.86$ & $5.21 \pm 1.11$ & 0.066 \\
\hline Triglyceride (mmol/L) & $1.48 \pm 1.28$ & $1.35 \pm 0.84$ & $1.39 \pm 1.02$ & 0.832 & & $1.30 \pm 0.82$ & $1.13 \pm 0.63$ & $1.20 \pm 0.79$ & 0.404 \\
\hline HDL-cholesterol (mmol/L) & $1.1 \pm 0.3$ & $1.2 \pm 0.3$ & $1.1 \pm 0.3$ & 0.458 & & $1.2 \pm 0.2$ & $1.2 \pm 0.3$ & $1.1 \pm 0.2$ & 0.635 \\
\hline LDL-cholesterol (mmol/L) & $3.15 \pm 0.76$ & $3.17 \pm 0.84$ & $3.11 \pm 0.70$ & 0.877 & & $3.39 \pm 0.83$ & $3.13 \pm 0.75$ & $3.32 \pm 0.84$ & 0.104 \\
\hline SGOT (U/L) & $25.3 \pm 7.2$ & $29.2 \pm 11.3$ & $26.4 \pm 7.0$ & 0.065 & & $25.9 \pm 8.0$ & $27.4 \pm 13.0$ & $29.7 \pm 11.8$ & 0.169 \\
\hline Creatinine ( $\mu \mathrm{mol} / \mathrm{L})$ & $81.3 \pm 23.0$ & $78.7 \pm 27.4$ & $76.9 \pm 14.1$ & 0.689 & & $81.3 \pm 22.1$ & $89.3 \pm 53.9$ & $81.3 \pm 19.4$ & 0.309 \\
\hline Uric acid (mmol/L) & $0.37 \pm 0.08$ & $0.38 \pm 0.08$ & $0.37 \pm 0.07$ & 0.568 & & $0.38 \pm 0.09$ & $0.40 \pm 0.11$ & $0.39 \pm 0.08$ & 0.756 \\
\hline Moderate exercise (\%) & 75.0 & 70.3 & 71.1 & 0.921 & & 87.5 & 72.1 & 68.5 & 0.134 \\
\hline Antihypertensive drug use (\%) & 25.0 & 31.2 & 16.1 & 0.107 & & 23.4 & 25.0 & 25.9 & 0.951 \\
\hline
\end{tabular}

Abbreviation: SGOT, serum glutamic oxaloacetic transferase.

Notes: Data are expressed as mean \pm SD. Comparisons between ACE genotype are performed by 1-way ANOVA or $\chi^{2}$-test.

$P$ : Data are adjusted by general linear models for age, sex, BMI, WHR, total cholesterol, HDL-cholesterol, use of antihypertensive drug, diabetes, and physical activity status. 


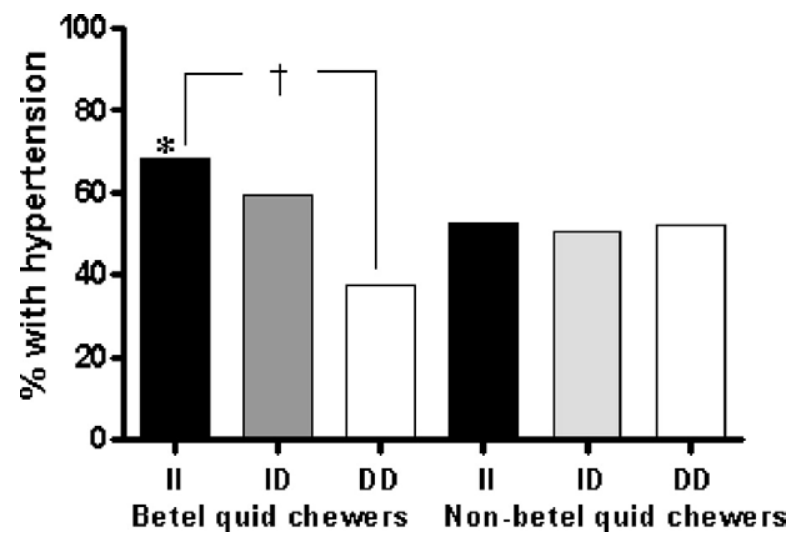

Fig 1. Prevalence of hypertension in study subjects stratified by $A C E$ genotypes and $\mathrm{BQ}$ chewing status. " $\mathrm{BQ}$ chewers with $A C E \mathrm{DD}$ genotype had a significantly lower hypertension rate than that of II genotype $(P<0.05)$ after adjusting by general linear models for age, sex, BMI, waist-to-hip ratio, total cholesterol, HDL-cholesterol, use of antihypertensive drugs, diabetes status, and physical activity status. ${ }^{\dagger} P_{\text {trend }}<0.05$. No statistical difference was found in the prevalence of hypertension among nonchewers with different $A C E \mathrm{I} / \mathrm{D}$ genotypes.

with the DD genotype who presented with lower SBP and prevalence of hypertension observed in the current study may indicate that BQ chewers with this genotype have greater hypotensive responses to the tannin substance contained in the areca nut than subjects with II or ID genotypes.

Areca nut extract has also been reported to induce higher basal secretion of catecholamines from adrenal chromaffin cells. ${ }^{33}$ However, data indicate that BQ chewing can affect cardiovascular function and that tolerance to the pressor response occurs in habitual users. ${ }^{13}$ The observation here reveals no significant difference in blood pressure measurements and prevalence of hypertension between controls and BQ users, which indicates that complex gene-environment factors exist in blood pressure homeostasis.

Johnston et $\mathrm{al}^{34}$ using rat brain slices demonstrated that BQ constituents arecholine and guvacoline hydrolyzed products arecaidine and guvacine, which act as substrate-competitive inhibitors of GABA uptake. Inhibition of GABA activity can promote an increase in appetite and is believed to be contributor to the pathogenesis of obesity in BQ chewers. ${ }^{5}$ The mechanism of $\mathrm{BQ}$ chewing associated with hyper-triglyceridemia is still unknown. Another issue that needs to be addressed is that the effect of the D allele, and BQ chewing seems to be restricted to SBP only. In accordance with our findings, Schut et $\mathrm{al}^{35}$ have also observed the smokingdependent effects of the $A C E$ I/D polymorphism on SBP and systolic hypertension and not on DBP. Findings regarding the $A C E \mathrm{I} / \mathrm{D}$ polymorphism and SBP or 
isolated systolic hypertension have not, however, been very conclusive as of yet. ${ }^{36,37}$

Several limitations to this study exist. As in all studies that rely on participant reports, recall bias and social desirability bias may have produced a spurious association. Furthermore, the sample size of this study remained small because of the strict selection criteria designed to exclude BQ chewers with smoking or alcohol usage. The definition of a nonchewer (ie, those who never consumed BQ in their lives) most likely included some people who were infrequent chewers. The inclusion of infrequent chewers in the nonchewer category causes the ORs for hypertension to be underestimated. Only the aborigines of Taiwan were studied, so caution is advised in generalizing these conditions to other populations.

In summary, the current data indicate that chronic BQ chewing may have diverse systemic effects, which suggests that a direct effect exists on blood pressure associated with BQ chewing and carrying of the D allele. It is hypothesized that synergistic effects on the regulation of blood pressure may explain the effects of the $\mathrm{D}$ allele and BQ chewing on SBP. Additional prospective and largescale studies are required to confirm the blood pressure effect of BQ consumption.

The authors are grateful to the staff of the Aboriginal Health Promotion Center and the health bureaus in the aboriginal communities for their assistance in the measurements and other organizational aspects of this study.

\section{REFERENCES}

1. Gupta PC, Ray CS. Epidemiology of betel quid usage. Ann Acad Med Singapore 2004;33:31-6.

2. Ko YC, Chiang TA, Chang SJ, Hsieh SF. Prevalence of betel quid chewing habit in Taiwan and related sociodemographic factors. J Oral Pathol Med 1992;21:261-4.

3. Yang YH, Lee HY, Tung S, Shieh TY. Epidemiological survey of oral submucous fibrosis and leukoplakia in aborigines of Taiwan. J Oral Pathol Med 2001;30:213-9.

4. Trivedy CR, Craig G, Warnakulasuriya S. The oral health consequences of chewing areca nut. Addict Bio 2002;7:115-25.

5. Boucher BJ, Mannan N. Metabolic effects of the consumption of Areca catechu. Addict Bio 2002;7:103-10.

6. Taylor RF, al-Jarad N, John LM, Conroy DM, Barnes NC. Betel-nut chewing and asthma. Lancet 1992;339:1134-6.

7. Benjamin AL. Community screening for diabetes in the National Capital District, Papua New Guinea: is betel nut chewing a risk factor for diabetes? P N G Med J 2001;44:101-7.

8. Sekkadde Kiyingi K, Saweri A. Betel nut chewing causes bronchoconstriction in some asthma patients. P N G Med J 1994;37:90-9.

9. Sharma AK, Gupta R, Gupta HP, Singh AK. Haemodynamic effects of pan masala in healthy volunteers. J Assoc Physicians India 2000;48:400-1.

10. Mannan N, Boucher BJ, Evans SJ. Increased waist size and weight in relation to consumption of Areca catechu (betel-nut); a risk factor for increased glycaemia in Asians in east London. Br J Nutr 2000;83:267-75.
11. Tung TH, Chiu YH, Chen LS, Wu HM, Boucher BJ, Chen TH Keelung Community-based Integrated Screening programme No. 2. A population-based study of the association between areca nut chewing and type 2 diabetes mellitus in men (Keelung Community-based Integrated Screening programme No. 2). Diabetologia 2004;47:1776-81.

12. Chung FM, Chang DM, Chen MP, Tsai JCR, Yang YH, Shieh TY, et al. Areca Nut Chewing is associated with metabolic syndrome. Role of tumor necrosis factor- $\alpha$, leptin, and white blood cell count in betel nut chewing-related metabolic derangements. Diabetes Care 2006;29:1714.

13. IARC Working Group. Tobacco habits other than smoking; betelquid and areca-nut chewing; and some related nitrosamines. Lyon, France, October 23-30, 1984. IARC Monogr Eval Carcinog Risk Chem Hum 1985;37:1-268.

14. Inokuchi J, Okabe H, Yamauchi T, Nagamatsu A, Nonaka G, Nishioka I. Antihypertensive substance in seeds of Areca catechu L. Life Sci 1986;38:1375-82.

15. Chu NS. Cardiovascular responses to betel chewing. J Formos Med Assoc 1993;92:835-7.

16. Weir MR, Dzau VJ. The renin-angiotensin-aldosterone system: a specific target for hypertension management. Am J Hypertens 1999;12:205S-13S

17. Rigat B, Hubert C, Alhenc-Gelas F, Cambien F, Corvol P, Soubrier F. An insertion/deletion polymorphism in the angiotensin I-converting enzyme gene accounting for half the variance of serum enzyme levels. J Clin Invest 1990;86:1343-6.

18. Li X, Du Y, Du Y, Huang X. Correlation of angiotensinconverting enzyme gene polymorphism with effect of antihypertensive therapy by Angiotensin-converting enzyme inhibitor J Cardiovasc Pharmacol Ther 2003;8:25-30.

19. Yu H, Zhang Y, Liu G. Relationship between polymorphism of the angiotensin-converting enzyme gene and the response to angiotensin-converting enzyme inhibition in hypertensive patients. Hypertens Res 2003;26:881-6.

20. Scharplatz M, Puhan MA, Steurer J, Bachmann LM. What is the impact of the $A C E$ gene insertion/deletion (I/D) polymorphism on the clinical effectiveness and adverse events of ACE inhibitors? Protocol of a systematic review. BMC Med Genet 2004;5:23-8.

21. Chung FM, Yang $\mathrm{YH}$, Chen $\mathrm{CH}$, Lin CC, Shieh TY Angiotensin-converting enzyme gene insertion/deletion polymorphism is associated with risk of oral precancerous lesion in betel quid chewers. Br J Cancer 2005;93:602-6.

22. Chung FM, Yang YH, Shieh TY, Shin SJ, Tsai JC, Lee YJ. Effect of alcohol consumption on estimated glomerular filtration rate and creatinine clearance rate. Nephrol Dial Transplant 2005; 20:1610-6

23. Chung FM, Tsai JC, Chang DM, Shin SJ, Lee YJ. Peripheral total and differential leukocyte count in diabetic nephropathy: the relationship of plasma leptin to leukocytosis. Diabetes Care 2005;28:1710-7.

24. Lee YJ, Tsai JCR. ACE gene insertion/deletion polymorphism associated with 1998 World Health Organization definition of metabolic syndrome in Chinese type 2 diabetic patients. Diabetes Care 2002;25:1002-8.

25. Shanmugam V, Sell KW, Saha BK. Mistyping ACE heterozygotes. PCR Methods Appl 1993;3:120-1.

26. Halkin A, Keren G. Potential indications for angiotensinconverting enzyme inhibitors in atherosclerotic vascular disease. Am J Med 2002;112:126-34

27. Tiret L, Rigat B, Visvikis S, Breda C, Corvol P, Cambien F, et al. Evidence, from combined segregation and linkage analysis, that a variant of the angiotensin I-converting enzyme 
(ACE) gene controls plasma ACE levels. Am J Hum Genet 1992;51:197-205.

28. Chiang FT, Lai ZP, Chern TH, Tseng CD, Hsu KL, Lo HM, et al. Lack of association of the angiotensin converting enzyme polymorphism with essential hypertension in a Chinese population. Am J Hypertens 1997;10:197-201.

29. Ding PY, Hu OY, Pool PE, Liao W. Does Chinese ethnicity affect the pharmacokinetics and pharmacodynamics of angiotensin-converting enzyme inhibitors? J Hum Hypertens 2000;14:163-70.

30. Baudin B. Angiotensin I-converting enzyme gene polymorphism and drug response. Clin Chem Lab Med 2000;38:853-6.

31. Ueda S, Meredith PA, Morton JJ, Connell JM, Elliott HL. ACE (I/D) genotype as a predictor of the magnitude and duration of the response an ACE inhibitor drug (enalaprilat) in humans. Circulation 1998;98:2148-53.

32. Stavroulakis GA, Makris TK, Krespi PG, Hatzizacharias AN, Gialeraki AE, Anastasiadis G, et al. Predicting response to chronic antihypertensive treatment with fosinopril: the role of angiotensin-converting enzyme gene polymorphism. Cardiovasc Drugs Ther 2000;14:427-32.
33. Wang CK, Hwang LS. Effect of betel quid on catecholamine secretion from adrenal chromaffin cells. Proc Natl Sci Counc Repub China B 1997;21:129-36.

34. Johnston GA, Krogsgaard-Larsen P, Stephanson A. Betel nut constituents as inhibitors of gamma-aminobutyric acid uptake. Nature 1975;258:627-8.

35. Schut AF, Sayed-Tabatabaei FA, Witteman JC, Avella AM, Vergeer JM, Pols HA, et al. Smoking-dependent effects of the angiotensin-converting enzyme gene insertion/deletion polymorphism on blood pressure. J Hypertens 2004;22:313-9.

36. Davis D, Liyou N, Johnson A. The ACE gene I/D polymorphism, but not the angiotensin II type I receptor gene A1166C polymorphism is associated with isolated systolic hypertension. $\mathrm{J}$ Hum Hypertens 2001;15:653-4.

37. Johnson AG, Simons LA, Friedlander Y, Simons J, Davis DR, MaCallum J. I/D polymorphism of the angiotensin-converting enzyme gene does not predict isolated systolic or systolicdiastolic hypertension in the elderly. J Hum Hypertens 1996; 10:167-9. 